\title{
sciendo
}

\section{Counterfactuals as Strict Conditionals}

\author{
Andrea Iacona \\ University of Turin \\ DOI: $10.2478 /$ disp-2015-0009 \\ BIBLID [0873-626X (2015) 41; pp. 165-191]
}

\begin{abstract}
This paper defends the thesis that counterfactuals are strict conditionals. Its purpose is to show that there is a coherent view according to which counterfactuals are strict conditionals whose antecedent is stated elliptically. Section 1 introduces the view. Section 2 outlines a reply to the main argument against the thesis that counterfactuals are strict conditionals. Section 3 compares the view with a proposal due to Åqvist, which may be regarded as its direct predecessor. Section 4 explains how the view differs from contextualist strict conditional accounts of counterfactuals. Finally, section 5 addresses the thorny issue of disjunctive antecedents.
\end{abstract}

\section{Keywords}

Counterfactuals, strict conditionals, modal logic, counterfactual fallacies, disjunctive antecedents.

\section{Ellipticism}

The line of thought that will be articulated in this paper rests on three basic assumptions. The first expresses a widely accepted idea about the meaning of counterfactuals. A counterfactual is a sentence 'If it were the case that $p$, then it would be the case that q', where ' $p$ ' and ' $q$ ' figure as the antecedent and the consequent. For example, the following sentence is a counterfactual:

(1) If kangaroos had no tails, they would topple over.

Its canonical formulation is 'If it were the case that kangaroos have no tails, then it would be the case that they topple over', where 'Kangaroos have no tails' is the antecedent and 'They topple over' is the consequent. The widely accepted idea is that the meaning of a counterfactual can be stated in terms of a quantification over possible

Disputatio, Vol. VII, No. 41, November 2015

Received: 24/07/2014 Revised: 22/02/2015 Accepted: 08/10/2015 
worlds restricted by a relation of similarity. As Lewis puts it,

'If kangaroos had no tails, they would topple over' seems to me to mean something like this: in any possible state of affairs in which kangaroos have no tails, and which resembles our actual state of affairs as much as kangaroos having no tails permits it to, the kangaroos topple over. ${ }^{1}$

More generally, if ' $p$-world' stands for a world in which ' $p$ ' is true, and 'the actual world' is used non-rigidly as an indexical expression that singles out the world of evaluation, the meaning of 'If it were the case that $p$, then it would be the case that $q$ ' may be stated as follows:

(M) In any $p$-world which is relevantly similar to the actual world, $q$.

The class of relevantly similar worlds may be characterized in different ways. One option, suggested by Stalnaker, it to say that there is a unique $p$-world most similar to the actual world. Another option, suggested by Lewis, is to say that there is a set of $p$-worlds most similar to the actual world. A third option, which will be adopted here, is to say that there is a set of $p$-worlds sufficiently similar to the actual world. The difference between 'most similar' and 'sufficiently similar' turns out clear in the case in which ' $p$ ' is true in the actual world. For in that case there is only one world most similar to the actual world, namely the actual world itself, while there may be more than one world sufficiently similar to the actual world. Anyway, this difference is not essential for the present purposes. What will be assumed is simply that, on any sensible view of counterfactuals, (M) provides a correct analysis of their meaning. ${ }^{2}$

The second assumption is that counterfactuals are context sensitive, in that they have different truth conditions in different contexts. Suppose that the following sentences are used to describe an imaginary situation in which Caesar is in command in Korea:

(2) If Caesar had been in command, he would have used the atom bomb.

${ }^{1}$ Lewis 1973: 1. The idea goes back at least to Leibniz 1985: 146-147.

${ }^{2}$ The difference considered can be framed in terms of the principles called Centering and Weak Centering, as explained in Arlo-Costa 2007, section 3.3. 
(3) If Caesar had been in command, he would have used catapults.

There is a sense in which (2) is true but (3) is false, and there is a sense in which (3) is true but (2) is false: in the first case one has in mind a modernized Caesar, while in the second one has in mind an unmodernized Caesar. This difference is plausibly described in terms of context sensitivity. In one context, we may attach more importance to similarities and differences of one kind, so that (2) turns out true, while in another context we may attach more importance to similarities and differences of another kind, so that (2) turns out false. The same goes for (3). More generally, a context can be defined as a set of parameters that includes a world $w$ and a selection function $f$ from sentence-world pairs to sets of worlds. For every sentence ' $p$ ', $f(p, w)$ is a set of $p$-worlds sufficiently similar to $w$, which means that $f$ determines both the weights with which similarities in particular respects contribute to overall similarity between worlds and what qualifies as a sufficient level of overall similarity. Assuming that the meaning of a counterfactual is given by $(\mathrm{M})$, different contexts may provide different interpretations of the expression 'relevantly similar to the actual world' which occurs in (M). This is to say that different contexts may determine different class of relevantly similar worlds.

The third assumption concerns logical form. To say that counterfactuals are strict conditionals is to say that they are sentences of the form $\square(\alpha \supset \beta)$. In the standard semantics of modal logic, $\square(\alpha \supset \beta)$ is true in a world $w$ if and only if $\alpha \supset \beta$ is true in every world accessible from $w$, that is, in every world that satisfies the restriction associated with the sort of necessity that $\square$ is intended to capture. What will be assumed here is that logical form is a matter of truth conditions: to say that a formula expresses the logical form of a sentence is to say that the formula provides a representation of the truth conditions of the sentence that can be employed in a formal explanation of its logical properties. The implications of this assumption turn out clear if one thinks that, given the second assumption, a principled distinction can be drawn between the meaning of a counterfactual and its truth conditions. While the meaning of a counterfactual is constant, its truth conditions may vary depending on context. So, if the formal representation of the counterfactual depends on its truth conditions, it must be sensitive to such variation. In other words, the 
primary sense in which a formula can be said to express the logical form of a counterfactual is that in which it represents the counterfactual as it is understood in a given context. Obviously, this assumption is not very orthodox. Most philosophers would be inclined to say that a counterfactual has a fixed logical form which is determined by its syntactic structure or by its meaning. But the issue of what is logical form cannot be addressed here. In what follows it will simply be taken for granted that the idea that logical form is a matter of truth conditions is interesting enough to deserve consideration.

Given these three assumptions, the thesis that counterfactuals are strict conditionals may be phrased as follows: for every counterfactual 'If it were the case that $p$, then it would be the case that $q$ ' and every context $c$, there is a formula of the form $\square(\alpha \supset \beta)$ which represents the truth conditions of the counterfactual as understood in $c$. More precisely, the view that will be considered entails that counterfactuals are strict conditionals whose antecedent is stated elliptically. On this view, which may be called ellipticism, 'If it were the case that $p$, then it would be the case that $q$ ', as uttered in $c$, is properly phrased as 'Necessarily, if $p$ and things are relevantly like in the actual world, then $q$ ', where the content of 'things are relevantly like in the actual world' is determined by $c$. Therefore, its logical form is $\square(\alpha \supset \beta)$, where $\alpha$ stands for ' $p$ and things are relevantly like in the actual world' as understood in $c$ and $\beta$ stands for ' $q$ '. In other words, $\alpha$ delimitates the set of worlds that the selection function of $c$ assigns to $p$ relative to the world of $c$. So the counterfactual can be represented as a strict conditional whose antecedent has two parts: one is explicit, 'p', the other is implicit, 'things are relevantly like in the actual world'.

According to ellipticism, the fact that a counterfactual may have different truth conditions in different contexts is representable at the formal level. Consider (2), and suppose that $c$ and $c^{\prime}$ are two contexts which differ in the way explained above. (2) is properly phrased as 'Necessarily, if Caesar is in command and things are relevantly like in the actual world, then he uses the atom bomb', where 'things are relevantly like in the actual world' has different contents in $c$ and $c^{\prime}$. Therefore, distinct formulas may be assigned to (2) relative to $c$ and $c^{\prime}$. That is, if (2) is represented as $\square(\alpha \supset \beta)$ relative to $c$, then it may be represented as $\square(\gamma \supset \beta)$ relative to $c^{\prime}$ : $\alpha$ stands for 'things are 
relevantly like in the actual world' as understood in $c$, while $\gamma$ stands for 'things are relevantly like in the actual world' as understood in $c$ '. This is consistent with a general principle about formalization that is usually taken for granted, namely, that sentences with different truth conditions must be represented by distinct formulas, that is, formulas that can have different truth values in the same model.

One way to see how the principle applies is to think about the difference between a counterfactual 'If it were the case that $p$, then it would be the case that $q$ ' and an overt strict conditional 'Necessarily, if $p$ then $q$ '. Consider the following sentence:

(4) Necessarily, if kangaroos have no tails, then they topple over. (1) and (4) have different truth conditions. For (4) means that kangaroos topple over in any possible world in which they have no tails. So if (1) and (4) were represented by the same formula, the difference between them would not be captured at the formal level. A straightforward way to draw the distinction is to assign different formulas to (1) and (4), that is, $\square(\alpha \supset \beta)$ and $\square(\gamma \supset \beta)$, where $\alpha$ stands for 'Kangaroos have no tails and things are relevantly like in the actual world' and $\gamma$ stands simply for 'Kangaroos have no tails'. This method of formalization implies that counterfactuals are covert strict conditionals. They differ from overt strict conditionals, whose antecedent is stated explicitly.

Note that, since counterfactuals and overt strict conditionals are represented by the same kind of formula, there is a clear sense in which they have the same logical form. The thesis that counterfactuals are strict conditionals, as understood here, is not intended to provide an analysis of the meaning of counterfactuals in terms of $\square$ and $\supset$. Counterfactuals exhibit distinctive semantic features that make them differ from other conditionals, and presumably there is no formula in the language of modal logic $-\square(\alpha \supset \beta)$ or any other - such that having a logical form expressed by that formula is both necessary and sufficient for having those features. Nonetheless, it may be claimed that counterfactuals are sentences of the form $\square(\alpha \supset \beta)$ in virtue of those features.

Ellipticism is essentially a view about the logical form of counterfactuals. Its main point concerns the formal representation of counterfactuals, rather than the analysis of their meaning. To illustrate 
this feature of ellipticism, consider the Stalnaker-Lewis view, that is, the shared fragment of the theories of counterfactuals defended by Stalnaker and Lewis. Ellipticism and the Stalnaker-Lewis view converge at the conceptual level, as they both rest on the idea that the meaning of a counterfactual is expressed by $(\mathrm{M})$. More precisely, in both cases a counterfactual 'If it were the case that $p$, then it would be the case that q' can be evaluated as true or false relative to a context defined in the way considered, provided that the selection function is appropriately specified. The key difference between the two views concerns the formal representation of counterfactuals. Stalnaker and Lewis think that a special symbol, say $>$, should be employed to capture the meaning of 'If it were the case that..., then it would be the case that...', hence that a special formal system that encompasses that symbol must be tailored to counterfactuals. According to ellipticism, instead, no logical adjustment of that kind is required. The only symbols needed are $\square$ and $\supset$, with their familar semantics. $^{3}$

\section{Counterfactual fallacies}

The main argument provided so far against the thesis that counterfactuals are strict conditionals is due to Stalnaker and Lewis. According to Stalnaker and Lewis, the thesis may appear tenable if one looks at a single counterfactual, but it proves inadequate if one reflects on sets of counterfactuals and the logical relations they involve. For at least three basic inference rules that hold for strict conditionals do not hold for counterfactuals, that is, there are at least three distinctive "counterfactual fallacies". The first is the fallacy of strengthening the antecedent. Consider the following argument:

A1 (5) If Otto had come, it would have been a lively party.

$\therefore$ (6) If Otto and Anna had come, it would have been a lively party.

Imagine that Otto is a very cheerful person, but that he just broke up

\footnotetext{
${ }^{3}$ In this respect, ellipticism differs from any attempt to define counterfactuals in terms of some characteristic modal operator analogous to $\square$, such as Burks 1951.
} 
with Anna after six months of endless rows. In such a situation (5) may be true even though (6) is false. In other words, (5) is consistent with

(7) If Otto and Anna had come, it would have been a dreary party. Therefore, A1 is invalid. But the following argument form is valid:

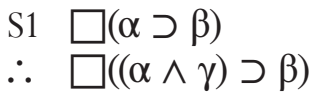

For if $\beta$ is true in all accessible $\alpha$-worlds, a fortiori it will be true in all accessible $\alpha$-worlds in which $\gamma$ is true. So A1 cannot instantiate S1. ${ }^{4}$

The second is the fallacy of transitivity. Consider the following argument:

A2 (8) If Otto had gone to the party, Anna would have gone.

(9) If Anna had gone to the party, Waldo would have gone.

$\therefore \quad$ (10) If Otto had gone to the party, Waldo would have gone.

Imagine that Waldo fancies Anna, although he never runs the risk of meeting his successful rival Otto. Imagine also that Otto was locked up at the time of the party, so that his going to the party is a remote possibility, but that Anna almost did go, as she hoped to meet him. In such a situation (8) and (9) may be true even though (10) is false. Therefore, A2 is invalid. However, the following argument form is valid:

\section{S2 $\square(\alpha \supset \beta)$ \\ $\square(\beta \supset \gamma)$ \\ $\therefore \square(\alpha \supset \gamma)$}

For if all accessible $\alpha$-worlds are $\beta$-worlds and all accessible $\beta$-worlds are $\gamma$-worlds, then all accessible $\alpha$-worlds are $\gamma$-worlds. So A2 cannot instantiate $\mathrm{S} 2 .^{5}$

${ }^{4}$ Stalnaker 1991: 38, Lewis 1973: 10-13 and 31. The sequence formed by (3) and (5) is called a "Sobel sequence", from Lewis 1973:10 fn.

${ }^{5}$ Stalnaker 1991: 38, Lewis 1973: 32-33. Note that S2 entails S1, as it is easily 
The third is the fallacy of contraposition. Consider the following argument:

A3 (11) If Otto had gone to the party, Anna would have gone.

$\therefore \quad$ (12)If Anna had not gone, Otto would not have gone.

Imagine that Otto wanted to go to the party but stayed away just to avoid Anna, while Anna would definitely have gone if Otto had been around. In such a situation (11) may be true even though (12) is false. Therefore, A3 is invalid. However, the following argument form, S3, is valid:

\section{S3 $\square(\alpha \supset \beta)$ \\ $\therefore \square(\sim \beta \supset \sim \alpha)$}

For $\alpha \supset \beta$ and $\sim \beta \supset \sim \alpha$ have the same truth value in every accessible world. So A3 cannot instantiate S3. ${ }^{6}$

The Stalnaker-Lewis argument may be summarized as follows. Suppose that counterfactuals are strict conditionals. Then A1-A3 instantiate S1-S3. But A1-A3 are invalid arguments, while S1-S3 are valid argument forms. Therefore, counterfactuals are not strict conditionals. Ellipticism provides a reason to reject this argument, as it undermines the assumption that if counterfactuals are strict conditionals then A1-A3 instantiate S1-S3. Consider A1. If (5) is represented as $\square(\alpha \supset \beta)$, then $\alpha$ does not stand for 'Otto has come' but for 'Otto has come and things are relevantly like in the actual world'. So (6) cannot be represented as $\square((\alpha \wedge \gamma) \supset \beta)$. For its whole antecedent is 'Otto and Anna have come and things are relevantly like in the actual world', in which neither conjunct amounts to $\alpha$. Therefore, the argument form instantiated by A1 is not S1 but the following:

$$
\begin{aligned}
& \text { S4 } \square(\alpha \supset \beta) \\
& \therefore \square(\gamma \supset \beta)
\end{aligned}
$$

seen if $\alpha$ is replaced with $\alpha \wedge \beta$. So the failure of S1 alone suffices to discard S2.

${ }^{6}$ Stalnaker 1991: 39, Lewis 1973: 35. 
Consider A2. If (8) is represented as $\square(\alpha \supset \beta)$, then (9) cannot be represented as $\square(\beta \supset \gamma)$ but rather as $\square(\gamma \supset \delta)$. Therefore, the argument form instantiated by $\mathrm{A} 2$ is not $\mathrm{S} 2$ but the following:

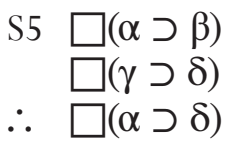

Finally, consider A3. If (11) is represented as $\square(\alpha \supset \beta)$, the antecedent of the formula that represents (12) cannot be $\sim \beta$ but a different formula $\gamma$. Similarly, its consequent cannot be $\sim \alpha$ but a different formula $\delta$ that stands for 'Otto has not gone'. Therefore, the argument form instantiated by $\mathrm{A} 3$ is not $\mathrm{S} 3$ but the following:

S6 $\square(\alpha \supset \beta)$

$\therefore \square(\gamma \supset \delta)$

Since S4-S6 are invalid forms, the invalidity of A1-A3 is easily explained.

Two final remarks. The first concerns the assumption that A1A3 are invalid. The ellipticist reply to the Stalnaker-Lewis argument grants this assumption: its point is that, even though A1-A3 are invalid, their invalidity is no evidence against the thesis that counterfactuals are strict conditionals. However, it is important to note that here it is not essential to assume that validity is a property of arguments, at least if arguments are understood in the usual way as sets of sentences. Perhaps the most plausible thing to say, given the contextsensitivity of counterfactuals, is that validity is a property of "interpreted" arguments, where an interpretation of an argument is an assignment of contexts to its sentences. If validity is so understood, the assumption to be granted is rather that A1-A 3 are invalid in the intended interpretation. This by itself does not rule out the possibility that there are interpretations in which A1-A3 or other structurally similar arguments are valid. For example, Lowe suggests that there are non-fallacious cases of transitivity:

Suppose that two people are discussing the influence of upbringing and social background upon a person's political convictions, and one 
of them, $X$, takes Margaret Thatcher as an example of someone who, though a firm supporter of the capitalist free market economy, might have had a quite different attitude towards it. $X$ reasons as follows:[...] If Margaret Thatcher had been born and brought up in the Soviet Union, she would have had communist sympathies; and if she had had communist sympathies, she would have been opposed to the capitalist free market economy. So, if she had been born and brought up in the Soviet Union, she would have been opposed to the capitalist free market economy. $X$ 's reasoning seems unexceptionable to the point of appearing almost banal. ${ }^{7}$

Lowe's example seems to show that an argument structurally similar to A2 can be valid in some interpretation. To see how this case differs from that of A2, it suffices to think that in this case, unlike in that of A2, the possible circumstances that one has in mind when one asserts the first premise also sustain the second premise. To put it another way, the possible circumstances that one has in mind when one asserts the first premise also justify strenghtening its consequent by adding the antecedent of the second premise as a conjunct. The following sentences seems equally assertable in the situation described:

(13) If Margaret Thatcher had been born and brought up in the Soviet Union, she would have had communist sympathies.

(14) If Margaret Thatcher had been born and brought up in the Soviet Union, she would have had communist sympathies and she had been opposed to the capitalist free market economy.

Obviously, this leaves open the question of how a principled distinction can be drawn between apparently fallacious cases and apparently non-fallacious cases. However, all that matters here is that ellipticism is consistent with the possibility that an argument structurally similar to A2 is valid in some interpretation, provided that no such argument is classified as a case of transitivity. S5 is an invalid argument form. But an invalid argument form can have valid instances.

The second remark concerns A3, which illustrates the difference between ellipticism and the Stalnaker-Lewis view explained in section 1. Stalnaker and Lewis claim that contraposition does not hold 
for counterfactuals: A3 is an invalid argument from $\alpha>\beta$ to $\sim \beta>$ $\sim \alpha$. Nonetheless, they maintain that modus tollens is a valid argument form. That is, from $\alpha>\beta$ and $\sim \beta$ we can infer $\sim \alpha$. This is why Lewis provides a non-orthodox justification of modus tollens that does not appeal to contraposition. He says that modus tollens is acceptable because from $\alpha>\beta$ we can infer $\alpha \supset \beta$, and from the latter we can infer $\sim \beta \supset \sim \alpha$ (contraposition does hold for $\supset$ ), so that $\sim \alpha$ follows by modus ponens. Even granting Lewis' justification, however, it is hard to resist our inclination to regard contraposition and modus tollens as different expressions of the same principle, and so to think that they should either stand or fall together. In a standard deduction system of modal logic, this inclination is vindicated by the rule of conditional proof: if one can derive $\beta$ from $\alpha$ and auxiliary premises, then one can derive $\alpha \supset \beta$ from the auxiliary premises alone. This means that if $\sim \alpha$ follows from $\sim \beta$ and $\alpha \supset \beta$, then $\sim \beta \supset \sim \alpha$ follows from $\alpha \supset \beta$. Ellipticism, unlike the Stalnaker-Lewis view, implies no separation between contraposition and modus tollens. According to ellipticism, both contraposition and modus tollens hold: arguments such as A3 simply have little to do with them. ${ }^{8}$

\section{The selection operator view}

In the past, some attempts have been made to provide an analysis of counterfactuals that employs the expressive resources of modal logic. Ellipticism bears close resemblance to one of them, due to Åqvist. According to Åqvist, counterfactuals can formally be represented in a modal language that contains an operator $*$ whose semantics is given in terms of a selection function $f$ that assigns sets of worlds to formulas. That is, $* \alpha$ is true in all and only the worlds that belong to $f(\alpha)$, where $f(\alpha)$ is understood as the set of $\alpha$-worlds most similar to the actual world. In such a language, the logical form of 'If it were the case that $p$, then it would be the case that $q$ ' may be expressed as $\square(* \alpha \supset \beta)$, where $\alpha$ stands for ' $p$ ' and $\beta$ stands for ' $q$ '. So it turns out that the counterfactual is true if and only $\beta$ is true in all $\alpha$-worlds

${ }^{8}$ Stalnaker 1991: 39, Lewis 1973: 36. This section is drawn from Iacona 2011. 
most similar to the actual world. ${ }^{9}$

Ellipticism has much in common with this view, which may be called the selection operator view. First, the central claim of both views is that the logical form of 'If it were the case that $p$, then it would be the case that $q$ ' is expressed by a strict conditional whose antecedent does not stand for ' $p$ ' but for a stronger condition that is implicit in the counterfactual. Second, both views assume that the implicit condition involves a similarity constraint in accordance with (M). Third, both views grant that the understanding of the similarity constraint may be irreducibly indexical, in that they do not require that the implicit condition amounts to a set of sentences whose conjunction provides a complete characterization of the set of worlds that satisfy the constraint. ${ }^{10}$

The obvious difference between ellipticism and the selection operator view is that ellipticism represents the whole antecedent of 'If it were the case that $p$, then it would be the case that q' as $\alpha$, so it requires no special symbol to be added to the language of modal logic. Given this difference, it is natural to wonder whether there are reasons to think that one of the two views is better than the other. One might be tempted to say that the selection operator view is preferable in that a representation that involves the operator $*$ displays a relation between the explicit part and the implicit part of the antecedent that a simple formula is unable to capture. But this temptation must be resisted. As it will be suggested, ellipticism is preferable in another respect, because it provides a neat account of some fundamental modal properties of counterfactuals that trouble the selection operator view. Therefore, all things considered it is not obvious that the selection operator view is better than ellipticism.

The selection operator view comes in at least two versions: one is the original version set out by Åqvist, the other is an amended version sketched by Lewis. Let us start with the original version. The semantics for $*$ provided by Åvist is rigidly centred on the actual world. Every model includes a distinguished world $w_{0}$, and the function $f$ is defined in terms of $w_{0}$ : the set that $f$ assigns to each formula

9 Åqvist 1973: 2-3.

${ }^{10}$ In this respect, both ellipticism and the selection operator view differ from what Lewis calls "the metalinguistic theory", see Lewis 1973: 66-67. 
$\alpha$ is understood as the set of $\alpha$-worlds most similar to $w_{0}$. However, as Lewis has argued, such a semantics is unable to account for the apparent contingency of some counterfactuals. Consider the following:

(15)If I had looked in my pocket, I would have found a coin.

Since I actually have a coin in my pocket, (15) is true in the actual world. But in a world in which my pocket is empty, (15) is false. This fact cannot be explained if $*$ is interpreted in the way considered. Certainly, if $\alpha$ stands for 'I looked in my pocket', which is the explicit part of the antecedent, then $* \alpha$ stands for 'I looked in my pocket and things are relevantly like in $w_{0}$ ', so the actual truth of (15) is explained in terms of the truth of $\square(* \alpha \supset \beta)$. But no explanation can be provided of the falsity of (15) in a world $w_{1}$ in which my pocket is empty. As Lewis observes, this is a serious limitation. Even if we are ultimately interested in the actual world, we must consider the truth values of counterfactuals at other worlds to obtain the actual truth values of sentences in which counterfactuals are embedded inside other counterfactuals. Consider the following:

(16)If I had looked in my pocket, I would have found a coin, but if my pocket were empty, it would not be the case that if I had looked in my pocket, I would have found a coin.

The actual truth of (16) can be explained only if the semantics makes room for the possibility that different sets of worlds are associated to the same antecedent. ${ }^{11}$

The amended version of the selection operator view is intended to make room for that possibility. As Lewis has explained, the view can be modified in order to account for the contingency of counterfactuals such as (15). His suggestion is that $f$ is replaced by a two-argument function $f^{\prime}$ that assigns sets of worlds to formula-world pairs, and that a three-place truth relation for $* \alpha$ is defined as follows: $* \alpha$ is true in a world $w$ with reference to a world $w^{\prime}$ if and only if $w$ belongs to $f^{\prime}\left(\alpha, w^{\prime}\right)$, that is, if and only if $w$ is one of the $\alpha$-worlds most similar to $w^{\prime}$. The three-place truth relation is then generalized to any formula by stipulating that the formula is true in $w$ with reference to $w^{\prime}$ if and only if it is true in $w$. So it turns out that $\square(* \alpha \supset \beta)$ is true in

${ }^{11}$ Lewis 1973: 61-62. 
${ }^{w}$ with reference to ${ }^{\prime}$ if and only if $* \alpha \supset \beta$ is true with reference to $w^{\prime}$ in every world accessible from $w$, that is, if and only if $\beta$ is true in every world in $f^{\prime}\left(\alpha, w^{\prime}\right)$ accessible from $w$. This way we get that there can be two worlds $w_{0}$ and $w_{1}$ such that $\square(* \alpha \supset \beta)$ is true in $w_{0}$ with reference to $w_{0}$, while it is false in $w_{1}$ with reference to $w_{1} \cdot{ }^{12}$

However, some important questions remain open. In the first place, it is not entirely clear how to make sense of the assumption that the intension of $* \alpha$ - the set of worlds in which $* \alpha$ is truevaries as a function of the world of reference. This assumption is intended to guarantee that the implicit part of the antecedent of a counterfactual can express different conditions relative to different worlds. To illustrate, let the meaning of (15) be stated as follows:

$\left(15_{M}\right)$ In any world in which I looked in my pocket, and in which things are relevantly like in the actual world, I found a coin.

A straightforward way to explain why (15) is true in the actual world $w_{0}$ but false in a world $w_{1}$ where my pocket is empty is to say that the implicit past of its antecedent expresses different conditions relative to $w_{0}$ and to $w_{1}$. This means that 'the actual world' in $\left(15_{M}\right)$ refers to $w_{0}$ in the first case and to $w_{1}$ in the second. That is,

$\left(15 w_{0}\right)$ Necessarily, if I looked in my pocket and things are relevantly like in $w_{0}$, then I found a coin.

$\left(15 w_{1}\right)$ Necessarily, if I looked in my pocket and things are relevantly like in $w_{1}$, then I found a coin.

The point, however, is that if the antecedent of a counterfactual expresses different conditions relative to different worlds, it is not clear why a single formula with variable intension should be used to represent those conditions. Note that the variation of intension at issue is not the familiar variation of intension due to a difference of model, but a variation of intension that occurs within a model. Of course, any standard semantics for a formal language allows that the same formula has different intensions in different models, given that models are normally understood as interpretations of the language. For example, the same formula can be read as 'Snow is white' in one case

${ }^{12}$ Lewis 1973: 62-63. 
and as 'Grass is green' in another case. But the variation of intension involved here is of a different kind, because it implies something like saying that the same formula, in the same model, is true in $w$ because it means 'Snow is white' relative to $w$ and false in $w$ ' because it means 'Grass is green' relative to $w^{\prime}$. This is quite an odd thing to say.

In the second place, Lewis does not explain how exactly the amended version of the selection operator view accounts for the actual truth of (16). In (16), two occurrences of the same antecedent hide different implicit conditions. The first condition concerns the actual world, while the second concerns a world in which my pocket is empty. Since both conditions are represented by $* \alpha,(16)$ must be formalized as a complex sentence in which $* \alpha$ occurs twice. Presumably, the sentence must be such that its truth in $w_{0}$ with reference to $w_{0}$ depends on the truth of the second occurrence of $* \alpha$ having a given intension relative to a different word $w_{1}$. However, the details of the account are still missing. Unless a formal semantics is spelled out with the due accuracy, it is hard to judge whether the problem has been solved.

In the third place, it may rightfully be asked whether the amended version of the selection operator view substantially preserves the thesis that counterfactuals are strict conditionals. If a counterfactual is represented by a formula $\square(* \alpha \supset \beta)$ that can be true in a world $w$ with reference to $w$ but false in a world $w^{\prime}$ with reference to $w^{\prime}$, then it can be treated as an ordinary contingent sentence. As Lewis suggests, if an operator $\dagger$ is so defined that $\dagger \alpha$ is true in $w$ if and only if $\alpha$ is true in $w$ with reference to $w$, we get that the counterfactual amounts to a contingent sentence of the form $\dagger \square(* \alpha \supset \beta)$. But a sentence of that form, it might be argued, is not a strict conditional. Independently of the presence of $\dagger$, the obvious difference is that a sentence of that form can be contingent, while a strict conditional must be necessary if it is true. For a strict conditional is a sentence of the form $\square \alpha$, and $\square \alpha$ entails $\square \square \alpha$. Or at least, this holds on the assumption that necessity obeys $\mathrm{S} 5$ or similar systems. ${ }^{13}$

From the foregoing considerations it turns out that it is not clear

\footnotetext{
${ }^{13}$ The operator $\dagger$ is introduced in Lewis 1973: 63. The point that strict conditionals must be necessary if they are true is made in Sider 2010: 200, where it is used against the thesis that counterfactuals are strict conditionals.
} 
how the selection operator view can cope with the issue of contingency. Ellipticism differs in this respect, in that it makes room for a distinction that explains how the apparent contingency of some counterfactuals squares with the thesis that counterfactuals are strict conditionals. According to ellipticism, there is a sense in which a counterfactual may be used to say the same thing in different worlds, and there is a sense in which it may be used to say different things in different worlds. The first is that in which the counterfactual has a meaning that does not vary from world to world, the meaning expressed by $(\mathrm{M})$. The second is that in which the counterfactual has different truth conditions in different worlds, given that the reference of the expression 'the actual world' which occurs in (M) varies from world to world. Since a context is a set of parameters which includes a world, this is to say that the reference of that expression may vary from context to context. In the first sense, the counterfactual may be contingent. In the second, it is necessary if true. For example, (15) may be used to say something true relative to $w_{0}$ and something false relative to $w_{1}$. But what is said relative to $w_{0}$, that is, $\left(15 w_{0}\right)$, is necessary if true. Similarly, what is said relative to $w_{1}$, that is, $\left(15 w_{1}\right)$, is impossible if false.

The contrast between ellipticism and the selection operator view emerges clearly if one considers the three questions raised above. In the first place, ellipticism does not need to assume that there are special formulas whose intension can vary within a model. In order to account for the fact that the antecedent of a counterfactual can express different conditions relative to different worlds, it is simply assumed that different conditions require different formulas. For example, $\left(15 w_{0}\right)$ and $\left(15 w_{1}\right)$ are represented as $\square(\alpha \supset \beta)$ and $\square(\gamma \supset \beta)$. Since 'things are relevantly like in the actual world' expresses different conditions relative to $w_{0}$ and $w_{1}$, different formulas $\alpha$ and $\gamma$ are used to represent those conditions. Therefore, the fact that (15) is true in $w_{0}$ but false in $w_{1}$ can be explained in terms of the platitude that different formulas may have different truth values.

In the second place, ellipticism can explain the actual truth of (16) in the same way. Since (16) contains two occurrences of 'I looked in my pocket' which are associated to different sets of worlds, these two occurrences are represented by different formulas, say $\alpha$ and $\gamma$. So the formalization of (16) does not require two occurences 
of $\alpha$. More generally, the method of formalization suggested is able to account for the semantic variation that affects the implicit part of the antecedent of counterfactuals, not only when this variation depends on the intended relations of similarity between worlds, but also when it depends on the world of utterance. This feature may pass unnoticed if one restricts attention to the truth values of counterfactuals in the actual world, but it becomes manifest when one considers the truth values that counterfactuals have in possible worlds different from ours.

In the third place, ellipticism definitely preserves the thesis that counterfactuals are strict conditionals. The understanding of the thesis suggested implies that strict conditionals are necessary if true. We saw that, although a counterfactual may be contingent in one sense, it may not in another sense. Since its formal representation as a strict conditional concerns the second sense, on the assumption that logical form is a matter of truth conditions, it turns out that the strict conditional must be necessary if true. Therefore, if a strict conditional is true in a world, its necessitation is also true in that world, in accordance with the S5 entailment from $\square \alpha$ to $\square \square \alpha$. For example, $\left(15 w_{0}\right)$ is formally represented as $\square(\alpha \supset \beta)$, where $\alpha$ stands for 'I looked in my pocket and things are relevantly like in $w_{0}$ '. So $\square(\alpha \supset \beta)$ expresses something about $w_{0}$ that is true in every world. This is why $\square \square(\alpha \supset \beta)$ is also true in $w_{0}$.

To sum up, the opposing inclinations towards contingency and necessity that emerge from the discussion of the selection operator view can be explained in terms of the distinction between meaning and truth conditions. Of course, one might still object that this distinction does not suffice, and insist that the intuition of contingency implies that the truth conditions of counterfactuals are themselves contingent. But nothing can be done to move such unsatisfied objector. First of all, the intuition of contingency, if there is such a thing, can hardly be so definite as to entail that it is not enough to say that the same sentence, with the same meaning, can be true in a world but false in another world. In the second place, it is reasonable to expect that a distinction along the lines suggested is the best that a strict conditional analysis can offer with respect to the issue of contingency. For a strict conditional analysis cannot rule out necessity altogether. As noted above, it would make little sense to claim that 
counterfactuals are sentences of the form $\square(\alpha \supset \beta)$ but deny that obeys S5 or similar systems. So the unsatisfied objector must think that in principle no strict conditional analysis can work.

\section{Contextualism}

Although it is generally taken for granted that counterfactuals are context sensitive, it is not entirely obvious to what extent they are context sensitive. One major point of controversy concerns the role of the antecedent in the determination of context. On the one hand, anyone agrees that the fact that different sets of worlds can be assigned to the same antecedent is correctly described in terms of context sensitivity. For example, it is plausible to say that (2) and (3) are true in different contexts, in that they are true relative to different ways of delimiting the class of relevantly similar worlds in which Caesar was in command. On the other hand, there is no equally shared account of the fact that, normally, different sets of worlds are assigned to different antecedents. For example, when (5) and (6) are evaluated respectively as true and false, the set of worlds that count as relevantly similar in the first case, those in which Otto has come to the party, differs from the set of worlds that count as relevantly similar in the second, those in which Otto and Anna have come to the party. The question, however, is whether this difference amounts to a difference of context: one option is to say that it does, the other is to say that it does not. ${ }^{14}$

The thesis that counterfactuals are strict conditionals is often associated with the first option. According to a line of thought that has been amply debated in the last few years, counterfactuals are highly context sensitive strict conditionals, in that their strictness varies as a function of their antecedent. Thus, (5) and (6) are strict conditionals assessed respectively as true and false in different contexts $c$ and $c^{\prime}$, that is, they involve different accessibility relations. The intended reading of (5) is that every accessible world in which Otto has come to the party is a world in which the party is lively. The intended reading of (6), instead, is that every accessible ${ }_{\mathbf{c}^{\prime}}$, world in which Otto and

${ }^{14}$ This question is explicitly addressed in Brogard and Salerno 2008, and in Cross 2011. 
Anna have come to the party is a world in which the party is lively. This means that A1 involves a context-shift, and the same goes for A2 and $\mathrm{A} 3 .^{15}$

If a strict conditional analysis of this kind is called contextualism, ellipticism differs from contextualism. Ellipticism rests on the assumption that counterfactuals are context sensitive in the less controversial sense, and contemplates no reason to think that they are context sensitive in the more controversial sense. As it turns out from section 1, a context may be defined in terms of a selection function. Consider two counterfactuals 'If it were the case that $p$, then it would be the case that $q$ ' and 'If it were the case that $r$, then it would be the case that $q$ ', and let $c$ be a context which includes a world $w$ and a selection function $f$. Since ' $p$ ' and ' $r$ ' are different sentences, $f(p, w)$ may differ from $f(r, w)$. But the context does not change, for $f$ is the same function. This turns out clear if the two counterfactuals are represented as $\square(\alpha \supset \beta)$ and $\square(\gamma \supset \beta)$, where $\alpha$ expresses an inclusion condition for $f(p, w)$ and $\gamma$ expresses an inclusion condition for $f(r, w)$. For such representation requires no variation in the accessibility relation: $\square$ expresses unrestricted necessity in both cases. In substance, ellipticism is a non-contextualist strict conditional analysis of counterfactuals. Its mere existence shows that the issue of how the context sensitivity of counterfactuals is to be understood must not be confused with the question of whether counterfactuals are strict conditionals.

Although an examination of the arguments that may be invoked to justify contextualism goes beyond the scope of this paper, at least one issue deserves attention. Contextualism, just like any strict conditional analysis, must provide a reply to the Stalnaker-Lewis argument. For that argument questions the thesis that counterfactuals are strict conditionals. However, it seems that none of the replies available to the advocates of contextualism is preferable to that outlined in section 2.

\footnotetext{
${ }^{15}$ The supposition that the counterfactuals in a Sobel sequence-hence in A1 - are strict conditionals that involve different contexts, initially dismissed in Lewis 1973, is developed in Von Fintel 2001 and in Gillies 2007. Similarly, Warmbrod (1981), Lowe (1990) and Lowe (1995) suggest that arguments such as A2 are affected by context-shifts, and Tichý (1984) says the same of arguments such as A3.
} 
The Stalnaker-Lewis argument is a reductio: the thesis that counterfactuals are strict conditionals is taken to entail the absurd consequence that A1-A3 instantiate S1-S3. Therefore, in order to reject the argument, it must be contended either that the thesis does not have the alleged consequence, or that the alleged consequence is not absurd. Perhaps the most natural option for the advocates of contextualism is the second. They might draw inspiration from Kaplan's treatment of arguments containing indexicals, and reply that it is wrong to assume that A1-A3 are invalid, for in order to assess A1A3, the context must be held fixed. According to Kaplan, an argument containing indexicals is valid if and only if, for any context, if the premises are true in that context, the conclusion must be true in that context. For example, 'She is there, so she is there' turns out valid on Kaplan's definition, because it can't be the case that a context makes 'She is there' true and false at the same time. A similar treatment may be applied to A1-A3: since validity amounts to truth preservation in any context, the fact that $\mathrm{A} 1-\mathrm{A} 3$ have true premises and false conclusion in the intended interpretation does not show that they are invalid, given that their intended interpretation involves context-shifts. ${ }^{16}$

This reply is not entirely satisfactory. If one assumes, following Kaplan, that validity is a property of arguments, and claim that A1-A3 are valid, despite the fact that their intended interpretation involves context-shifts, one has a straightforward account of the relation between A1-A3 and S1-S3: A1-A3 are valid in that they instantiate S1-S3. The obvious drawback of this reply, however, is that it clashes with the apparent invalidity of A1-A3 in the intended interpretation. Kaplan's definition leaves unexplained the fact that A1-A3 can be used in such a way that their premises are true and their conclusion is false, just as it leaves unexplained the fact that 'She is there, so she is there' can be used in such a way that its premise is true and its conclusion is false. If an argument is valid, one may be tempted to say, how can it be the case that its premises are true and its conclusion false? As it has been argued against Kaplan, a definition

\footnotetext{
${ }^{16}$ Kaplan's definition is suggested in Kaplan 1989. A reasoning along the lines considered is offered in Lowe 1990 and in Brogaard and Salerno 2008, although it is not accompanied by a strict conditional analysis of counterfactuals.
} 
of validity that holds for arguments containing context sensitive expressions should take into account non-univocal interpretations of their premises and conclusions, that is, interpretations which involve context-shifts. ${ }^{17}$

A different way to question the assumption that A1-A3 are invalid is to assume that validity is a property of interpreted arguments, and claim that, although A1-A3 are invalid in the intended interpretation, they are valid in other interpretations, so it is wrong to say that they are invalid simpliciter. The advantage of this reply is that it accounts for the apparent invalidity of A1-A3 in the intended interpretation. Its disadvantage, however, is that the relation between A1-A3 and S1-S3 becomes problematic. On the standard understanding of formal validity, an argument form is valid if and only if all its instances are valid. Assuming that validity is a property of interpreted arguments, this is to say that an argument form is valid if and only if all its instances are valid interpreted arguments. But then it turns out that some valid argument forms, S1-S3, have invalid instances, which is quite hard to accept. ${ }^{18}$

What has been said so far shows that it is not clear how the advocates of contextualism can reject the assumption that A1-A3 are invalid. Of course, rejecting that assumption is not the only way to deny the absurdity of the alleged consequence that A1-A3 instantiate $\mathrm{S} 1-\mathrm{S} 3$. The other way is to reject the assumption that $\mathrm{S} 1-\mathrm{S} 3$ are valid. However, such a reply throws the baby out with the bathwater. To say that S1-S3 are invalid is to deny the basic principles of modal logic. For the validity of S1-S3 follows from those principles. If $\mathrm{S} 1-\mathrm{S} 3$ are invalid, then the semantics of the language in which they are expressed is not the familiar semantics of modal logic, and $\square$ does not have its familiar meaning. Even if one is willing to accept this consequence, which is not easy to swallow, the question remains of how the thesis that counterfactuals are strict conditionals can be maintained in some sense that matters to the Stalnaker-Lewis argument. For that argument is intended to establish that counterfactuals

${ }^{17}$ This line of argument is developed in different ways in Yagisawa 1993, Iacona 2010, and Georgi 2015.

${ }^{18}$ Note that the case of a valid form with invalid instances significantly differs from the case considered in section 2 of an invalid form with valid instances. 
aren't strict conditionals just in the familiar sense.

Since the advocates of contextualism can hardly deny the absurdity of the alleged consequence of the thesis that counterfactuals are strict conditionals, it seems that a better option for them is to deny that the thesis has that consequence. As it turns out from section 2, this is the kind of reply provided by ellipticism. However, there are significant differences at the formal level. If counterfactulas are strict conditionals whose strictness varies as a function of their explicit antecedent, the obvious way to formally represent their variablity is to adopt indexed necessity operators $\square_{i}$, where each $i$ bears some relation to the antecedent of the formula in which it occurs. This way it can be contended that A1-A3 do not instantiate S1-S3 but invalid schemas in which different indices occur. Although there is nothing intrinsically wrong with this option, its formal part need be developed in order to be properly assessed, as it departs to some extent from standard modal logic. Ellipticism implies nothing like that, since S1-S3 are replaced by invalid schemas in the same language, S4-S5. So it seems that the best reply to the Stalnaker-Lewis argument that the advocates of contextualism can offer is a logically more complex variant of the ellipticist reply.

\section{Disjunctive antecedents}

This last section shows how ellipticism can handle the old problem of disjunctive antecedents. The problem concerns the inference schema called simplification of disjunctive antecedents, or SDA:

SDA If $p$ or $q$ had been the case, then $r$ would have been the case. $\therefore \quad$ If $p$ had been the case, then $r$ would have been the case.

On the one hand, it may seem that SDA is a valid schema, for there are clear cases in which we reason in accordance with it. Consider the following sentence:

(17)If either Oswald had not fired or Kennedy had been in a bullet-proof car, Kennedy would be alive today.

What (17) conveys is that each of two possible events, Oswald not firing and Kennedy being in a bullet-proof car, would have lead to 
the same result independently of the other, Kennedy being alive today. So it seems that from (17) we can infer

(18)If Oswald had not fired, Kennedy would be alive today.

And the same goes for the other disjunct. ${ }^{19}$

On the other hand, it has been argued that SDA is invalid, in that there are clear counterexamples to it. Suppose someone asks which side Spain fought on in World War II, and we reply that Spain did not enter the war, then adding the following sentence:

(19)If Spain had fought on the Axis side or on the Allied side, she would have fought on the Axis side.

In this case what we definitely are not willing to infer

(20) If Spain had fought on the Allies side, she would have fought on the Axis side. ${ }^{20}$

When uttering (19), we don't want to say that each of two possible events, Spain fighting on the Axis side and Spain fighting on the Allies side, would have lead to the same result independently of the other, Spain fighting on the Axis side. Rather, we want to say that if the disjunction 'Spain fought on the Axis side or Spain fought on the Allied side' were true, it would be true in virtue of the first disjunct. Therefore, not every counterfactual 'If $p$ or $q$ had been the case, then $r$ would have been the case' is like (17).

According to the Stalnaker-Lewis view, SDA is invalid. If one represents the premise as $(\alpha \vee \beta)>\gamma$ and the conclusion as $\alpha>\gamma$, one gets an invalid argument form: it may be the case that $(\alpha \vee \beta)>$ $\gamma$ is true, because every relevantly similar $\beta$-world is a $\gamma$-world, while $\alpha>\gamma$ is false. The friends of the Stalnaker-Lewis view have provided at least two arguments against the validity of SDA. The first goes as follows. Inferences such as that from (17) to (18) are indeed plausible. But their plausibility can be explained without assuming that SDA is a valid schema. Although it might seem that (17) has the form $(\alpha \vee \beta)>\gamma$, in reality it has the form $(\alpha>\gamma) \wedge(\beta>\gamma)$, hence (18) amounts to one of its conjuncts. The word 'or' in (17) is not to be

${ }^{19}$ Fine 1975:453, Nute 1975:775-776, Ellis, Jackson and Pargetter 1977:355.

${ }^{20}$ The example comes from McKay and Van Inwagen 1977. 
read in the standard way, as it often happens. Sometimes the surface structure of natural language is misleading. ${ }^{21}$

This argument is not very convincing. It is legitimate to suppose that the plausibility of the inference from (17) to (18) can be explained without assuming that SDA is a valid schema. But the claim that (17) has the form $(\alpha>\gamma) \wedge(\beta>\gamma)$ requires an independent justification, and it is not clear that such a justification can be provided. The trouble is not only the weakness of the evidence for that claim, but also the strength of the evidence against it. As it has been observed, 'or' seems to behave in the usual way when negated. Consider the following sentence:

(21) If it had not been the case that either Oswald had not fired or Kennedy had been in a bullet-proof car, Kennedy would not be alive today.

Prima facie, (21) is equivalent to 'If it had been the case that Oswald had fired and Kennedy had not been in a bullet-proof car, Kennedy would not be alive today'. This is exactly what we should expect given the standard assumption that $\sim(\alpha \vee \beta)$ is equivalent to $\sim \alpha \wedge$ $\sim \beta$. Instead, if the logical form of (17) were $(\alpha>\gamma) \wedge(\beta>\gamma)$, its logical form would be something like $\sim((\alpha>\sim \gamma) \wedge(\beta>\sim \gamma))$ or $\sim(\alpha>\sim \gamma) \wedge \sim(\beta>\sim \gamma)$, which is quite implausible. ${ }^{22}$

The second argument is that the assumption that SDA is a valid schema, combined with the apparently innocuous principle of substitution of equivalents, leads to undesirable results. Since $\alpha$ is equivalent to $(\alpha \wedge \beta) \vee(\alpha \wedge \sim \beta)$, by substitution of equivalents we get that $\alpha>\gamma$ entails $((\alpha \wedge \beta) \vee(\alpha \wedge \sim \beta))>\gamma$. But if SDA is a valid schema, from $((\alpha \wedge \beta) \vee(\alpha \wedge \sim \beta))>\gamma$ we get $(\alpha \wedge \beta)>\gamma$. So it turns out that $\alpha>\gamma$ entails $(\alpha \wedge \beta)>\gamma$, which is the unacceptable rule of strengthening the antecedent. ${ }^{23}$

This argument can have some effect only on those who accept the formalization suggested by the Stalnaker-Lewis view, hence reject the thesis that counterfactuals are strict conditionals. For if the

${ }^{21}$ Loewer 1976: 534-537, McKay and Van Inwagen 1977: 355, Lewis 1977 : 360-361.

${ }^{22}$ See Ellis, Jackson and Pargetter 1977: 356.

${ }^{23}$ Fine 1975: 453, Lewis 1977: 359. 
thesis holds, no such trouble can arise. According to a strict conditional analysis, the most natural formal counterpart of SDA is a valid argument form:

S7 $\square((\alpha \vee \beta) \supset \gamma)$

$\therefore \square(\alpha \supset \gamma)$

Since every $\alpha$-world is a $\alpha \vee \beta$-world, if every accessible $\alpha \vee \beta$-world is a $\gamma$-world, every accessible $\alpha$-world must be a $\gamma$-world. Assuming substitution of equivalents, from S7 we get that $\square(\alpha \supset \gamma)$ entails $\square((\alpha \wedge \beta) \supset \gamma)$. But there is nothing wrong with that, since S1 is valid.

From the two arguments considered emerges no straightforward solution to the problem of disjunctive antecedents. It is reasonable to say that SDA is not a valid schema, in that not every sentence that may occur as a premise of SDA is like (17). Undoubtedly, a distinction must be drawn between counterfactuals such as (17) and counterfactuals such as (19). But it would be nice to have an explanation of this distinction that does not rest on highly debatable assumptions. Ellipticism can provide such explanation.

Consider (17). In this case it is said that if each of the disjuncts that occur in the antecedent were true, it would make the consequent true. Accordingly, (17) is properly phrased as follows: necessarily, if Oswald has fired and things are relevantly like in the actual world or Kennedy has been in a bullet-proof car and things are relevantly like in the actual world, then Kennedy is alive today. So its formal representation is $\square((\alpha \vee \beta) \supset \gamma)$, where $\alpha$ stands for 'Oswald has fired and things are relevantly like in the actual world', and $\beta$ stands for 'Kennedy has been in a bullet-proof car and things are relevantly like in the actual world'. Since the logical form of (18) is $\square(\alpha \supset \gamma)$, (17) entails (18) in virtue of S7.

Now consider (19). In this case it is said that if the disjunction that forms the antecedent were true, the consequent would make it true. Accordingly, (19) is properly phrased as follows: necessarily, if Spain fought either on the Axis side or on the Allied side and things are relevantly like in the actual world, then Spain fought on the Axis side. So its formal representation is $\square(\alpha \supset \beta)$, where $\alpha$ stands for 'Spain fought either on the Axis side or on the Allied side and things 
are relevantly like in the actual world', and $\beta$ stands for 'Spain fought on the Axis side'. Since the formal representation of (20) is $\square(\gamma \supset$ $\beta$ ), where $\gamma$ stands for 'Spain fought on the Allies side and things are relevantly like in the actual world', the inference from (19) to (20) is not formally valid.

More generally, SDA is ambiguous. There are cases of SDA in which the premise is adequately represented as a strict conditional with a disjunctive antecedent, and cases of SDA in which the premise does not have that form. The inferences of the first kind are valid because they instantiate S7. Those of the second kind are invalid because they instantiate an invalid argument form.

This explanation, just like that proposed by the friends of the Stalnaker-Lewis view, implies that there is no strict rule for the formalization of a sentence 'If $p$ or $q$ had been the case, then $r$ would have been the case'. The recipe adopted so far for counterfactuals whose antecedent is a simple sentence or a conjunction works for (19) but not for (17). However, the account of (17) suggested entails no drastic revision of its apparent structure. This turns out clear if we consider the relation between (17) and (21). If (17) is represented as $\square((\alpha \vee \beta) \supset \gamma)$, there is no trouble with the negation of its explicit antecedent. (21) can be represented as $\square(\sim(\alpha \vee \beta) \supset \sim \gamma)$, which is equivalent to $\square((\sim \alpha \wedge \sim \beta) \supset \sim \gamma)$ on the usual understanding of 'or'. This means that the logical form of (17) and the logical form of (21) turn out to be related exactly in the way one would expect.

Andrea Iacona Center for Logic, Language, and Cognition Department of Philosophy and Education University of Turin

Via S. Ottavio 20, 10124 Turin andrea.iacona@unito.it

\section{References}

Åqvist, L. 1973. Modal Logic with Subjunctive Conditionals and Dispositional Predicates. Journal of Philosophical Logic 2: 1-76.

Arlo-Costa, H. 2007. The Logic of Conditionals, The Stanford Encyclopedia of Philosophy (Summer 2014 Edition), Edward N. Zalta (ed.), URL $=<$ http:// plato.stanford.edu/archives/sum2014/entries/logic-conditionals/>. 
Brogaard, B. and Salerno, J. 2008. Counterfactuals and Context. Analysis 68: $39-46$.

Burks, A. W. 1951. The Logic of Causal Propositions. Mind 60: 363-382.

Cross, C. B. 2011. Comparative World Similarity and What is Held Fixed in Counterfactulas. Analysis 71: 91-96.

Ellis, B.; Jackson, F. and Pargetter, R. 1977. An Objection to Possible-World Semantics for Counterfactual Logic. Journal of Philosophical Logic 6: 355-357.

Fine, K. 1975. Critical notice, 'Counterfactuals'. Mind 84: 451-458.

Georgi, G. 2015. Logic for Languages Containing Referentially Promiscuous Expressions. Journal of Philosophical Logic 44: 429-451.

Gillies, A. S. 2007. Counterfactual Scorekeeping. Linguistics and Philosophy 30: 329-360.

Iacona, A. 2010. Truth Preservation in any Context. American Philosophical Quarterly 47: 191-199.

Iacona, A. 2011. Counterfactual Fallacies. Humana.Mente 19 :1-9.

Kaplan, D. 1989. Demonstratives. In Themes from Kaplan, ed. by J. Perry J. Almog and H. Wettstein. Oxford University Press, 481-563.

Leibniz, G. W. 1985. Theodicy. Open Court.

Lewis, D. 1973. Counterfactuals. Blackwell.

Lewis, D. 1977. Possible World Semantics for Counterfactual Logics: A rejoinder. Journal of Philosophical Logic 6: 359-363.

Loewer, B. 1976. Counterfactuals with Disjunctive Antecedents. Journal of Philosophy 73: 531-537.

Lowe, E. J. 1990. Conditionals, Context, and Transitivity. Analysis 50: 80-87.

Lowe, E. J. 1995. The Truth about Counterfactuals. Philosophical Quarterly 45: 41-59.

McKay, T. and Van Inwagen, P. 1977. Counterfactuals with Disjunctive Antecedents. Philosophical Studies 31: 353-356.

Nute, D. 1975. Counterfactuals and the Similarity of Worlds. Journal of Philosophy 72: 773-778.

Sider, T. 2010. Logic for Philosophy. Oxford University Press.

Stalnaker, R. 1991. A Theory of Conditionals. In Conditionals, ed. by F. Jackson. Oxford University Press, 28-45.

Tichý, P. 1984. Two Parameters vs. Three. Philosophical Studies 45: 147-179.

von Fintel, K. 2001. Counterfactuals in a Dynamic Context. In A Life in Language, ed. by M. Kenstowicz and Ken Hale. MIT Press, 123-152.

Warmbrod, K. 1981. Counterfactuals and Substitution of Equivalent Antecedents. Journal of Philosophical Logic 10: 267-289.

Yagisawa, T. 1993. Logic Purified. Nous 27: 470-486. 